Pure and Applied Mathematics Quarterly

Volume 3, Number 2

(Special Issue: In honor of

Leon Simon, Part 1 of 2)

$615-630,2007$

\title{
Sobolev Critical Exponents of Rational Homotopy Groups
}

\author{
Tristan Rivière \\ Dedicated to Robert Gulliverto Robert Hardt and to \\ Leon Simon on the occasion of their 60th birthdays
}

\begin{abstract}
A closed simply connected manifold $N^{n}$, an integer $p$ and an element $z \in\left(\pi_{p}\left(N^{n}\right) \otimes \mathbb{Q}\right)^{*}$ being given, we define the algebraic and the analytic Sobolev critical exponent $\nu_{z}$ and $\mu_{z}$ of $z$. The algebraic Sobolev critical exponent $\nu_{z}$ of $z$ is related to the minimal number of branchs needed in trees representing $z$ using the minimal model construction of Sullivan . The analytic Sobolev critical exponent $\mu_{z}$ of $z$ is related to the logarithmic increase of the minimal $W^{1, p}$ norm $\|u\|_{W^{1, p}}$ among the maps $u$ from the $p$-sphere $S^{p}$ into $N^{n}$ as $z(u)$ goes to $+\infty$. We study the relation between $\nu_{z}$ and $\mu_{z}$ and we describe the crucial role played by these exponents in the problem of approximating Sobolev maps between manifolds by smooth maps. These exponents and their connection with the approximation problem in Sobolev Spaces were originally introduced by Robert Hardt and the author in [HR3].
\end{abstract}

\section{The strong approximation problem by Smooth maps in Sobolev SPACES BETWEEN MANIFOLDS.}

let $N^{n}$ be a submanifold of an euclidian space $\mathbb{R}^{k}$ and denote $B^{m}$ the unit ball in $\mathbb{R}^{m}$ centered at 0 . For $p \geq 1$ we define the Sobolev space

$$
W^{1, p}\left(B^{m}, N^{n}\right):=\left\{u \in W^{1, p}\left(B^{m}, \mathbb{R}^{k}\right) \text { s. t. } u(x) \in N^{n} \text { for a. e. } x \in B^{m}\right\}
$$

where $W^{1, p}\left(B^{m}, \mathbb{R}^{k}\right)$ is the usual notation for the measurable maps from $B^{m}$ into $\mathbb{R}^{k}$ which are in $L^{p}$ and whose distributional gradient is also in $L^{p}$. The strong approximation problem has to do with the following question :

Received Apr 13, 2006. 
Question : Are smooth maps from $B^{m}$ into $N^{n}$ dense in $W^{1, p}\left(B^{m}, N^{n}\right)$ for the $W^{1, p}$ norm?

A quite complete answer to this question is given by the following result.

Theorem I.1. [Wh], [ScU], [BeZ], [Bet1] $C^{\infty}\left(B^{m}, N^{n}\right)$ is dense in $W^{1, p}\left(B^{m}, N^{n}\right)$ if and only if

$$
p \geq m
$$

or

$$
\pi_{[p]}\left(N^{n}\right)=0
$$

where $[p]$ denotes the largest integer less or equal to $p$.

For instance, the map which assign to $x$ in the unit ball $B^{3}$ it's orthogonal nearest projection $x /|x|$ on the boundary $\partial B^{3} \simeq S^{2}$ is in the Sobolev space $W^{1,2}\left(B^{3}, S^{2}\right)$ but, as we will explain below, it cannot be approximated by smooth maps in $C^{\infty}\left(B^{3}, S^{2}\right)$ with respect to the $W^{1,2}$ norm.

Remark I.1. If $B^{m}$ is replaced by a more general riemannian manifold $\left(M^{m}, h\right)$ theorem I.1 happens not to be true anymore and the necessary and sufficient conditions for having the density of smooth maps is more complex. It is described in [HaL1], [HaL2] and [HaL3] by F.Hang and F.H.Lin (see also the discussion and the notion of local and global obstructions to the strong approximation in [HR2]).

The obstruction to the strong density of $C^{\infty}\left(B^{m}, N\right)$ in $W^{1, p}\left(B^{m}, N\right)$.

Let $p$ be an integer. The following theorem by B.White permits to extend the notion of homotopy classes to arbitrary maps in $W^{1, p}\left(S^{p}, N\right)$.

Theorem I.2. [Wh] (Existence of homotopy classes in $W^{1, p}\left(S^{p}, N^{n}\right)$ ) Let $p \in \mathbb{N}$ and $g \in W^{1, p}\left(S^{p}, N^{n}\right)$, then there exists $\varepsilon>0$ such that the following holds : Let $h$ and $k$ be two arbitrary maps in $C^{\infty}\left(S^{p}, N\right)$

$$
\left.\begin{array}{l}
\|h-g\|_{W^{1, p}}<\varepsilon \\
\|k-g\|_{W^{1, p}}<\varepsilon
\end{array}\right\} \Longrightarrow \quad h \text { is homotopic to } k
$$

Since $C^{\infty}\left(S^{p}, N\right)$ is dense in $W^{1, p}\left(S^{p}, N\right)$ (from theorem I.1) we can then define, without ambiguity, the homotopy class in $\pi_{p}(N)$ of an arbitrary map $g$ in $W^{1, p}\left(S^{p}, N\right)$ as being the homotopy class of any smooth map in $C^{\infty}\left(S^{p}, N\right)$ close enough to $g$. Such a class will be denoted $[g]$

Remark I.2. What was used in [Wh] is the fact that any map in $W^{1, p}\left(S^{p}, N\right)$ belongs to the space VMO - maps of Vanishing Mean Oscillations - this is a direct consequence of the Poincaré inequality. The VMO property is really the one which permits to extend the notion of homotopy class. This fact was later rediscovered in $[\mathrm{BrN} 1]$ and $[\mathrm{BrN} 2]$. 
Granting this result of White, and taking now $p \in \mathbb{R}$ and a map $u$ in $W^{1, p}\left(B^{[p]+1}\right.$, $N)$, using Fubini theorem, the restriction $u \mid \partial B_{r}^{[p]+1}(a)$ of $u$ to the boundary $\partial B_{r}^{[p]+1}(a)$ of the ball of center $a$ and radius $r$ is in $W^{1, p}$ for any $a \in B^{[p]+1}$ and almost every $r$ between 0 and $1-|a|$. Therefore the class

$$
\left[u_{\partial B_{r}^{[p]+1}(a)}\right] \in \pi_{[p]}(N)
$$

is well defined for every $a$ in $B^{[p]+1}$ and almost every $r$ in $(0,1-|a|)$. We then have the following characterisation of the approximability of a map $u$ in $W^{1, p}\left(B^{[p]+1}, N\right)$ by smooth maps

Theorem I.3. [Bet1] Let $p \in \mathbb{R}, p \geq 1$. Let $u \in W^{1, p}\left(B^{[p]+1}, N\right)$ there exists a sequence of smooth maps in $C^{\infty}\left(B^{[p]+1}, N\right)$ converging to $u$ in $W^{1, p}$ if and only if the following holds

$$
\forall a \in B^{[p]+1}(0) \quad, \quad \text { for a.e. } r \in(0,1-|a|) \quad\left[u_{\left.\right|_{\partial B_{r}^{[p]+1}(a)}}\right]=0
$$

More generally, for $m>[p]+1$, the same result holds for $\partial B_{r}^{[p]+1}(a)$ being replaced by generic $[p]$-spheres in $B^{m}$. Heuristically, what this result says, is that the lack of approximability of maps in $W^{1, p}\left(B^{m}, N\right)$ comes from realization on a "full measure" of $[p]$-sphere of non trivial homotopy class in $\pi_{[p]}(N)$.

Going back now to our previous example, the map $u$ from $B^{3}$ into $S^{2}$ assigning to $x$ the projection $x /|x|$, we observe that the restriction of $u$ to the boundary of every ball containing 0 has a topological degree equal to +1 and is therefore non homotopic to 0. Comparing this observation with theorem I.3 we obtain the fact that $u$ cannot be approximated by smooth maps in $C^{\infty}\left(B^{3}, S^{2}\right)$ with respect to the $W^{1,2}$ norm. This can be explained more directly in the following way : assuming there would exists a sequence $u_{i}$ in $C^{\infty}\left(B^{3}, S^{2}\right)$ converging to $u$ in $W^{1,2}$, then, using a standard argument based on the use of Fubini theorem and the mean value formula, for almost every $r \in(0,1)$, there exists a subsequence $u_{i^{\prime}}$ converging strongly to $u$ in $W^{1,2}\left(\partial B_{r}(0), S^{2}\right)$. Using now theorem I.3 with $g$ being the restriction of $u$ to $\partial B_{r}(0), h$ being the restriction of $u_{i^{\prime}}$ to $\partial B_{r}(0)$ and $k$ being also the restriction of $u$ to $\partial B_{r}(0)$, we would get that, for $i^{\prime}$ large enough, $u$ is homotopic to $u_{i^{\prime}}$ on $\partial B_{r}(0)$. But the restriction of $u_{i^{\prime}}$ to $\partial B_{r}(0)$ admits a smooth extension in $C^{\infty}\left(B_{r}(0), S^{2}\right)$ which is $u_{i^{\prime}}$ itself, thus the restriction of $u_{i^{\prime}}$ to $\partial B_{r}(0)$ is null-homotopic. This would then imply that the restriction of $u$ to $\partial B_{r}(0)$ which is equal to $r^{-1} x$ should also be null homotopic which is a contradiction.

This last example illustrates perfectly the mechanism behind the impossibility of approximating some maps in $W^{1, p}\left(B^{m}, N\right)$ by maps in $C^{\infty}\left(B^{m}, N\right)$ when 
$\pi_{[p]}(N)$ is not trivial. We could then say the the strong approximation issues in

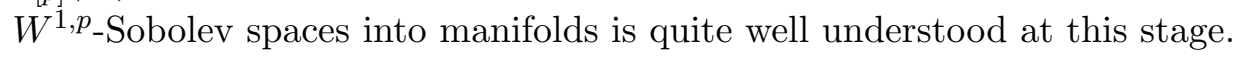

\section{The Weak approximation Problem by Smooth maps in SoboleV SPACES BETWEEN MANIFOLDS.}

We consider now a closed submanifold $N^{n}$ of some euclidian space $\mathbb{R}^{k}$ and an exponent $p \geq 1$ such that $\pi_{[p]}\left(N^{n}\right) \neq 0$ and a dimension $m>[p]$. Let $u$ be a map in $W^{1, p}\left(B^{m}, N^{n}\right)$ which is not in the closure of $C^{\infty}\left(B^{m}, N^{n}\right)$ for the $W^{1, p}$ strong topology (existence of such a $u$ is given by theorem I.1), the weak approximation problem has to do with the following question

Question : Does there exist a sequence $u_{i}$ in $C^{\infty}\left(B^{m}, N\right)$ which weakly converge to $u$ in $W^{1, p}$ ?

It is not too difficult to see that a positive answer to that question for every $u$ in $W^{1, p}\left(B^{m}, N^{n}\right)$ would require at least that $p$ is an integer (i.e. $\left.p=[p]\right)$. This comes from the fact that on generic $[p]$-sphere on which the restriction of the map $u$, that we are trying to weakly approximate, is non null homotopic. Thus a sequence of smooth maps $u_{n}$ converging weakly to $u$ would have to "jump" in homotopy classes at the limit in order to converge to $u$. This would happen only by point concentrations on the sphere due to the concentration compactness theory (see $[\mathrm{SaU}]$ ). Point concentration is tolerated by the $W^{1, p}$ energy (does not make this energy blow-up) for $p \geq[p]$ only if $p$ equals to the dimension of the sphere $[p]$ (the homogeneous $W^{1, p}$-pseudo-norm is then conformal invariant). Thus, from now on untill the end of the paper, we will make the following assumption :

i)

ii)

$$
p=[p] \in \mathbb{N},
$$

iii)

$$
m>p \quad,
$$

$$
\pi_{p}(N) \neq 0
$$

Known answers to the weak approximation problem by smooth maps in Sobolev spaces

In fact there are very few answers to the above question which seems to be quite challenging. At this stage of developpement of our knowledge not a single map is known, in any of the $W^{1, p}\left(B^{m}, N\right)$, for any choice of $p \in \mathbb{N}, m>p$ and $N^{n}$, which cannot be approximated weakly in $W^{1, p}$ by smooth maps in $C^{\infty}\left(B^{m}, N\right)$. Here are the known answers : 
Theorem II.1. [BBC], [ABL] Let $p \in \mathbb{N}, m \in \mathbb{N}$ and let $u$ in $W^{1, p}\left(B^{m}, S^{p}\right)$ then there exists a sequence $u_{i}$ in $C^{\infty}\left(B^{m}, S^{p}\right)$ such that $u_{i}$ converges weakly to $u$ in $W^{1, p}$.

Theorem II.2. [Haj] Let $N^{n}$ be a p-1-connected closed submanifold of some euclidian space $\mathbb{R}^{k}\left(\pi_{l}\left(N^{n}\right)=0\right.$ for $\left.1 \leq l \leq p-1\right)$, let $m \in \mathbb{N}$ and let $u$ in $W^{1, p}\left(B^{m}, N^{n}\right)$ then there exists a sequence $u_{i}$ in $C^{\infty}\left(B^{m}, N^{n}\right)$ such that $u_{i}$ converges weakly to $u$ in $W^{1, p}$.

This result can be seen as an extension of the previous one in the sence that the $p$-1-connectedness assumption of $N^{n}$ implies that the cell decomposition of $N^{n}$ starts at most with a bouquet of $p$-spheres and the difficulties reduce more or less to the ones already tackled while proving theorem II.1.

The case of the exponent $p=2$ is completely answered in the following result

Theorem II.3. $[\mathrm{PaR}]$ Let $N^{n}$ be a closed submanifold of some euclidian space $\mathbb{R}^{k}$, let $m \in \mathbb{N}$ and let $u$ in $W^{1,2}\left(B^{m}, N^{n}\right)$ then there exists a sequence $u_{i}$ in $C^{\infty}\left(B^{m}, N^{n}\right)$ such that $u_{i}$ converges weakly to $u$ in $W^{1,2}$.

In the case where $N^{n}$ is simply connected the previous result is already contained in theorem II.2. The new difficulty while proving theorem II.3 is to handle non trivial $\pi_{1}(N)$. Since the $\pi_{2}$ of the one dimensional skeleton of $N^{n}$ is zero, this case has also some relevance to the two previous results theorem II.1 and theorem II.2

Finally we have the following result

Theorem II.4. [HR4] Let $p>2$, let $m \in \mathbb{N}$ and let $u$ in $W^{1, p+1}\left(B^{m}, S^{p}\right)$ then there exists a sequence $u_{i}$ in $C^{\infty}\left(B^{m}, S^{p}\right)$ such that $u_{i}$ converges weakly to $u$ in $W^{1, p+1}$.

Here the obstruction to the strong approximation comes from the $\pi_{p+1}\left(S^{p}\right)$ (and not the $\pi_{p}\left(S^{p}\right)$ anymore) which is stable and equal to $\mathbb{Z}_{2}$ for $p>2$. In this sense this result is very different in nature form the previous theorems II.1...II.3. The proof is using an innovative technic based on Tom-Pontryagin characterization of stable homotopy groups and seems to give good hope to handle other cases where the obstruction to the strong approximation comes from finite stable homotopy groups.

There are some other results quite related to the one we just mentioned above : see [Ri2], [Han], [Pak], [GiM].

We illustrate now the question of the weak approximability by smooth maps by presenting one of the simplest situation, generic however, solved by theorem II.1 : the case $W^{1,2}\left(B^{3}, S^{2}\right)$. So we present an answer to the following question 
Question : Why is $C^{\infty}\left(B^{3}, S^{2}\right)$ weakly sequentially dense in $W^{1,2}\left(B^{3}, S^{2}\right)$ ?

Let $u$ be an element in $W^{1,2}\left(B^{3}, S^{2}\right)$ which is not approximable by smooth maps in $C^{\infty}\left(B^{3}, S^{2}\right)$. We assume to simplify the presentation that the trace of the map $u$ on $\partial B^{3}$ is constant. The obstruction to the strong approximability comes in the present situation from the homotopy group $\pi_{2}\left(S^{2}\right)=\mathbb{Z}$. It was observed in [Bet1] that, even if $u$ cannot be approximated by smooth maps, $u$ can be however approximated by maps which are smooth away from finitely many points. Precisely we introduce the space

$$
\mathcal{R}_{1,2}^{\infty}\left(B^{3}, S^{2}\right)=\left\{v \in C^{\infty}\left(B^{3} \backslash K, S^{2}\right) \cap W^{1,2} \text { where } K \text { is a finite set in } B^{3}\right\}
$$

(observe that $K$ depends a-priori on $v$ ). Then there exists $v_{i}$ in $\mathcal{R}_{1,2}^{\infty}$ which strongly converge to $u$ in $W^{1,2}$. It is also possible to make $\mathcal{R}_{1,2}^{\infty}$ a bit smaller by requiring that the homotopy class of $u$ on each sphere, with small enough radius, centered on each point in $K$ is not trivial. Denote $K_{i}=\left\{a_{j_{1}} \cdots a_{j_{Q_{i}}}\right\}$ the set of points where $v_{i}$ is not smooth and denote $d_{j_{1}} \cdots d_{j_{Q_{i}}}$ the homotopy group (the topological degree in $\mathbb{Z}$ ) of the restriction of $u$ to any sphere (having a small enough radius) centered respectively at $a_{j_{1}} \cdots a_{j_{Q_{i}}}$. The idea is to remove these points by doing some surgery on the map $v_{i}$ which do not modify $v_{i}$ on the "main part" of $B^{3}$ and which hopefully would not increase "too much" the energy. Precisely, we first connect the $a_{j_{l}}$ according to their multiplicity $d_{j_{l}}$ in an optimal way : we are using a 1-dimensional de Rham current $\mathbb{L}_{i}$ corresponding to the integration of 1 form along a union of oriented straight segments $\mathcal{L}_{i}$ with integer multiplicity $\theta$ and $\mathbb{L}_{i}$ is chosen in such a way that it minimizes the mass $M\left(\mathbb{L}_{i}\right)$ under the constraint

$$
\partial \mathbb{L}=\sum_{l=1}^{Q_{i}} d_{j_{l}} \delta_{a_{j_{l}}}
$$

Then, a small positive number $\varepsilon$ being given, we modify $v_{i}$ in the $\varepsilon$-neighborhood $U_{\varepsilon}$ of the support of $\mathbb{L}_{i}$ given by $U_{\varepsilon}=\{x ; \operatorname{dist}(x, \operatorname{supp} \mathbb{L})<\varepsilon\}$. We remove $v_{i}$ in $U_{\varepsilon}$ and we insert instead of $v_{i}$, all along the support of $\mathbb{L}$, a covering of $S^{2}$, according to the multiplicity of $\mathbb{L}_{i}$, in order to anihilate the degree of $v_{i}$ at each $a_{j_{l}}$ (see [Bet2] and figure II). 


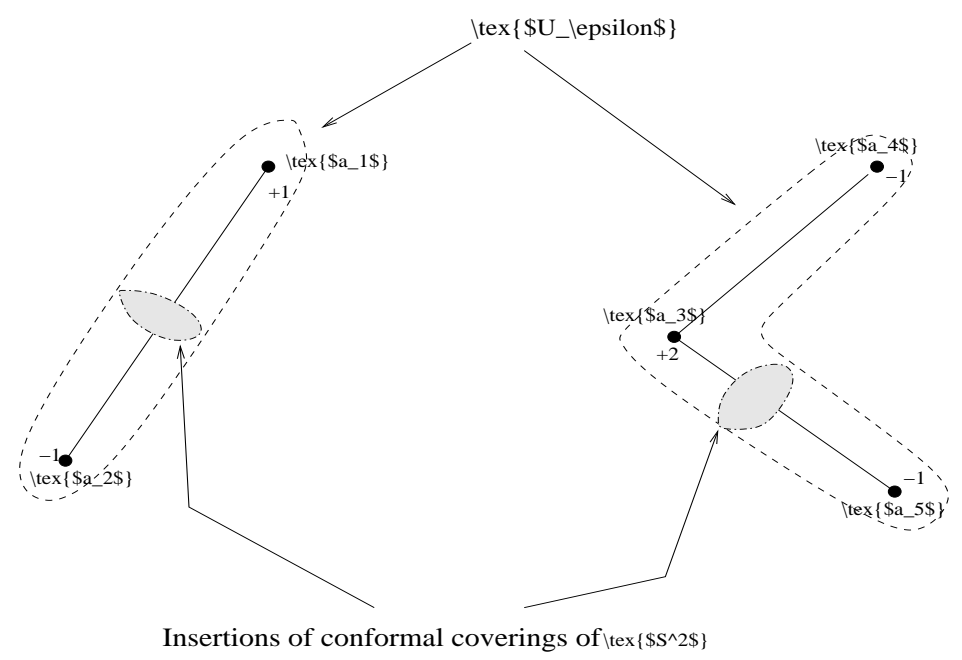

Figure 1. The surgery realized on the map $v_{i}$ in order to remove the topological singularities $a_{j_{l}}$.

The new map that we obtain after this surgery will be denoted $v_{i}^{\varepsilon}$. It is possible to do so without modifying $v_{i}$ outside $U_{\varepsilon}$ and with an energy control of the form

$$
\int_{B^{3}}\left|\nabla v_{i}^{\varepsilon}\right|^{2} \leq \int_{B^{3}}\left|\nabla v_{i}\right|^{2}+8 \pi M\left(\mathbb{L}_{i}\right)+\delta_{i}(\varepsilon)
$$

where $\delta_{i}(\varepsilon)$ goes to zero as $\varepsilon$ converges to 0 . Since $v_{i}^{\varepsilon}$ and $v_{i}$ are bounded in $L^{\infty}$, since they coincide on the whole ball $B^{3}$ except on $U_{\varepsilon}$, a set of measure of order $O\left(\varepsilon M\left(\mathbb{L}_{i}\right)\right)$ and since $v_{i}$ converges strongly to $u$ in $W^{1,2}$, it is now clear that, taking $\varepsilon$ small enough for each $i$, the sequence $v_{i}^{\varepsilon}$ converges to $u$ in every $L^{p}$ for $p<+\infty$. In order then to ensure that this convergence is a weak convergence in $W^{1,2}$ it remains to bound the $W^{1,2}$ energy of $v_{i}^{\varepsilon}$. In view of (II.1), it suffices then to bound $M\left(\mathbb{L}_{i}\right)$ uniformly in $i$. The following result provide a control to $M\left(\mathbb{L}_{i}\right)$ and is the key point in the proof. Precisely we have

Theorem II.5. [BCL] Let $v$ be a map in $\mathcal{R}_{1,2}^{\infty}\left(B^{3}, S^{2}\right)$, constant on $\partial B^{3}$, then there exists a union of oriented segments $\mathcal{L}$ and an "integer valued multiplicity map" theta on $\mathcal{L}$ (i.e. a measurable map $\theta: \mathcal{L} \rightarrow \mathbb{Z}$ ) such that

$$
\partial \mathbb{L}=\sum_{l=1}^{Q_{i}} d_{j_{l}} \delta_{a_{j_{l}}}
$$

where $a_{j_{l}}$ are the point singularities of $v, d_{j_{l}}$ is the topological degree of the restriction of $v$ to small spheres surrounding $a_{j_{l}}$ and $\mathbb{L}$ is the following current

$$
\forall \psi \in C_{0}^{\infty}\left(B^{3}, \wedge^{1} \mathbb{R}^{3}\right) \quad\langle\mathbb{L} ; \psi\rangle=\int_{\mathcal{L}} \theta \psi .
$$


There exists such a $\mathbb{L}$ for which the following bound holds

$$
M(\mathbb{L})=\int_{\mathcal{L}}|\theta| d \mathcal{H}^{1} \leq \frac{1}{8 \pi} \int_{B^{3}}|\nabla v|^{2}
$$

Combining then this result and estimate (II.1), choosing $\varepsilon=\varepsilon_{i}$ small enough for each $i$, we have obtained a sequence $u_{i}:=v_{i}^{\varepsilon_{i}}$ converging weakly to $u$ in $W^{1,2}$.

\section{Connecting topological singular sets : the $\pi_{2}\left(S^{2}\right)$ case}

As we just saw the main issue in proving the weak sequential density of smooth maps in $W^{1,2}\left(B^{3}, S^{2}\right)$ was the control of the mass of the current $\mathbb{L}$ "connecting" the point singularities $a_{j}$ according to their multiplicity $d_{j}$ of an arbitrary map $v$ in $\mathcal{R}_{1,2}^{\infty}\left(B^{3}, S^{2}\right)$ by the mean of it's $W^{1,2}$ energy. The current $\sum_{j} d_{j} \delta_{a_{j}}$ is the obstruction to strongly approximate $v$ by smooth maps in $C^{\infty}\left(B^{3}, S^{2}\right)$ and is what we call the topological singular set of $v$ as we saw in theorem I.3(see the definition of the topological singular sets in general situations in [HR2]). This current can be obtained in the present situation by the mean of the following computation : let $\omega$ be a 2 -form on $S^{2}$ whose integral on $S^{2}$ equals to 1 , denote $v^{*} \omega$ the pull-back on $B^{3}$ of $\omega$ by $v$, then the following holds

$$
d\left(v^{*} \omega\right)=\sum_{l=1}^{Q} d_{l} \delta_{a_{l}} .
$$

Taking now an arbitrary map $u$ in $W^{1,2}\left(B^{3}, S^{2}\right)$ the distribution $d\left(u^{*} \omega\right)$ still make sense and characterizes the possibility for $u$ to be in the strong closure of smooth maps for the $W^{1,2}$ norm. Precisely it is proved in [BCDH] that there exists $u_{i}$ in $C^{\infty}\left(B^{3}, S^{2}\right)$ converging strongly to $u$ in $W^{1,2}$ if and only if

$$
d\left(u^{*} \omega\right)=0 .
$$

For that reason and the fact that for every $a \in B^{3}$ and for almost every $r>0$

$$
\int_{B_{r}^{3}(a)} d\left(u^{*} \omega\right):=\int_{\partial B_{r}^{3}(a)} u^{*} \omega=\left[u_{\left.\right|_{\partial B_{r}^{3}(a)}}\right] \in \pi_{2}\left(S^{2}\right) \simeq \mathbb{Z}
$$

the distribution $d\left(u^{*} \omega\right)$ is also called the topological singular set of $u$. It was proved in [GMS] that is possible to extend theorem II.5 to general maps in $W^{1,2}\left(B^{3}, S^{2}\right)$. Denoting $\mathcal{H}^{1}$ the 1 -dimensional Hausdorf measure, we have the following result.

Theorem II.6. [GMS] Let $u$ be in $W^{1,2}\left(B^{3}, S^{2}\right)$ constant on the boundary, then there exist a countable union $\mathcal{L}=\cup_{j} \mathcal{L}_{j}$ of disjoint $\mathcal{H}^{1}$-measurable subsets of $C^{1}$ oriented curves and an $\mathcal{H}^{1}$ measurable "integer valued multiplicity map" $\theta$ from $\mathcal{L}$ into $\mathbb{Z}$ satisfying

$$
\int_{\mathcal{L}}|\theta| d \mathcal{H}^{1} \leq \frac{1}{8 \pi} \int_{B^{3}}|\nabla u|^{2}
$$


and for every $a$ in $B^{3}$ and almost every $r \in(0,1-|a|)$

$$
\left[u_{\left.\right|_{\partial B_{r}(a)}}\right]=\sum_{x_{0} \in \partial B_{r}(a) \cap \mathcal{L}} \theta\left(x_{0}\right) \operatorname{sgn}\left\{\vec{l}\left(x_{0}\right) \cdot\left(x_{0}-a\right)\right\}
$$

where $\vec{l}$ is the tangent direction to the $C^{1}$ curve containing $x_{0}$ and the corresponding $\mathcal{L}_{j}$ in $\mathcal{L}$.

Using the language of Geometric Measure Theory (see [Fe]), this theorem gives the existence of a 1-dimensional integer rectifiable current $\mathbb{L}$ (which is the integration on $\mathcal{L}$ according to the multiplicity $\theta$ ) satisfying

$$
\partial \mathbb{L}=d\left(u^{*} \omega\right),
$$

(observe that (II.6) is equivalent to (II.5)) and such that the mass of $\mathbb{L}$ is bounded by $(8 \pi)^{-1} \int|\nabla u|^{2}$.

\section{Connecting topological singular sets : the $\pi_{3}\left(S^{2}\right)$ case}

We are now looking at the situation where the obstruction to the strong approximation comes from the homotopy group $\pi_{3}\left(S^{2}\right)$, which is equal also to $\mathbb{Z}$. Thus we take the target to be again $N=S^{2}$, but the exponent is now $p=3$ and the smallest $m$ such that ii) above is satisfied : $m=4$. This means that we are looking at the space $W^{1,3}\left(B^{4}, S^{2}\right)$. Although it looks quite similar to the previous situation -replacing formally the topological degree (class in $\pi_{2}\left(S^{2}\right)$ ) by the Hopf topological degree (class in $\pi_{3}\left(S^{2}\right)$ )- this case is unsolved yet : the following question is still open.

Open Problem : Let $u$ be in $W^{1,3}\left(B^{4}, S^{2}\right)$, does there exist $u_{i}$ in $C^{\infty}\left(B^{4}, S^{2}\right)$ converging weakly to $u$ in $W^{1,3}$ ?

In trying to adapt the approach which was succesful to tackle the $\pi_{2}\left(S^{2}\right)$ case we introduce the space

$$
\mathcal{R}_{1,3}^{\infty}\left(B^{4}, S^{2}\right)=\left\{v \in C^{\infty}\left(B^{4} \backslash K, S^{2}\right) \cap W^{1,3} \text { where } K \text { is a finite set in } B^{4}\right\}
$$

Like in the previous case this space is dense for the strong topology in $W^{1,3}\left(B^{4}, S^{2}\right)$. Similarly as above we can even consider a smaller space by requiring that the homotopy class in $\pi_{3}\left(S^{2}\right)$ of the restriction of $v$ to small spheres centered at each singularity in $K$ is non trivial : it's Hopf degree is non zero.

Given then an arbitrary $u$ in $W^{1,3}\left(B^{4}, S^{2}\right)$ and a sequence $v_{i}$ in $\mathcal{R}_{1,3}^{\infty}\left(B^{4}, S^{2}\right)$ converging strongly to $u$, the same surgery work will produce, for every $\varepsilon$ small enough, a familly of maps $v_{i}^{\varepsilon}$ in $C^{\infty}\left(B^{4}, S^{2}\right)$ coinciding with $v_{i}$ outside an $\varepsilon$ neighborhood $U_{\varepsilon}$ of a union of segments connecting the point singularities $a_{j_{l}}$ of $v_{i}$, taking into account the multiplicity $d_{j_{l}}$ given by the class in $\pi_{3}\left(S^{2}\right)$ realized by 
the restriction of $v_{j}$ to $\partial B_{r}\left(a_{j_{l}}\right)$. This is again possible with the following control of the $W^{1,3}$ energy

$$
\int_{B^{4}}\left|\nabla v_{i}^{\varepsilon}\right|^{3} \leq \int_{B^{4}}\left|\nabla v_{i}\right|^{3}+C_{0} M\left(\mathbb{L}_{i}\right)+\delta_{i}(\varepsilon)
$$

where $\delta_{i}(\varepsilon)$ tends to zero as $\varepsilon$ goes to zero and $M\left(\mathbb{L}_{i}\right)$ is the mass of the current realized by the integration along these segments connecting the point singularities $a_{j_{l}}$ according to their multiplicities : $\partial \mathbb{L}_{i}=\sum_{l} d_{j_{l}} \delta_{a_{j_{l}}}$. In order to be now able to conclude the proof of the existence of a sequence of smooth maps converging weakly to $u$ we would need to find a control of the mass of the minimal connection $\mathbb{L}_{i}$ by the mean of the 3 -energy of $v_{i}$ which strongly converge to $v$. In contrast with the previous case such a control does not exists and there is no corresponding phenomenon to the one observed in the $B^{3}-S^{2}$ case in theorem II.5. Precisely we have.

Theorem II.7. [HR1] There exists $u$ in $W^{1,3}\left(B^{4}, S^{2}\right)$ such that for sequence every $v_{i}$ in $\mathcal{R}_{1,3}^{\infty}\left(B^{4}, S^{2}\right)$ converging strongly to $u$ in $W^{1,3}$ the following holds

$$
\min \left\{M\left(\mathbb{L}_{i}\right) \quad ; \quad \partial \mathbb{L}_{i}=\sum_{l=1}^{Q_{i}} d_{j_{l}} \delta_{a_{j_{l}}}\right\} \longrightarrow+\infty \quad
$$

where $a_{j_{l}}$ are the point singularities of $v_{i}$ and $d_{j_{l}}$ the topological Hopf degree of $v_{i}$ about these points (i.e. the class of $\left[v_{i} \mid \partial B_{r}\left(a_{j_{l}}\right)\right]$ in $\pi_{3}\left(S^{2}\right)$ for small $r$ ).

In fact the $u$ constructed in theorem II.7 satisfies the following

Theorem II.8. [HR1] There exists $u$ in $W^{1,3}\left(B^{4}, S^{2}\right)$ constant on the boundary such that for every countable union $\mathcal{L}=\cup_{j} \mathcal{L}_{j}$ of disjoint $\mathcal{H}^{1}$-measurable subsets of $C^{1}$ oriented curves and an $\mathcal{H}^{1}$ measurable "integer valued multiplicity map" $\theta$ from $\mathcal{L}$ into $\mathbb{Z}$ satisfying for every a in $B^{4}(0)$ and almost every $r \in(0,1-|a|)$

$$
\left[u_{\left.\right|_{\partial B_{r}(a)}}\right]=\sum_{x_{0} \in \partial B_{r}(a) \cap \mathcal{L}} \theta\left(x_{0}\right) \operatorname{sgn}\left\{\vec{l}\left(x_{0}\right) \cdot\left(x_{0}-a\right)\right\}
$$

where $\vec{l}$ is the tangent direction to the $C^{1}$ curve containing $x_{0}$ and the corresponding $\mathcal{L}_{j}$ in $\mathcal{L}$, then

$$
\int_{\mathcal{L}}|\theta| d \mathcal{H}^{1}=+\infty
$$

Roughly speaking, countrary to the $B^{3}-S^{2}$ case, there exist maps in $W^{1,3}\left(B^{4}, S^{2}\right)$ whose topological singular set cannot be "connected" by a finite mass rectifiable current. However the following holds

Theorem II.9. [HR1] Let u be a map in $W^{1,3}\left(B^{4}, S^{2}\right)$ constant on the boundary. $u$ can be approximated weakly in $W^{1,3}$ by smooth maps in $C^{\infty}\left(B^{4}, S^{2}\right)$ if and only if there exists a countable union $\mathcal{L}=\cup_{j} \mathcal{L}_{j}$ of disjoint $\mathcal{H}^{1}$-measurable subsets of 
$C^{1}$ oriented curves and an $\mathcal{H}^{1}$ measurable "integer valued multiplicity map" $\theta$ from $\mathcal{L}$ into $\mathbb{Z}$ satisfying for every $a$ in $B^{4}$ and almost every $r \in(0,1-|a|)$

$$
\left[u_{\left.\right|_{\partial B_{r}(a)}}\right]=\sum_{x_{0} \in \partial B_{r}(a) \cap \mathcal{L}} \theta\left(x_{0}\right) \operatorname{sgn}\left\{\vec{l}\left(x_{0}\right) \cdot\left(x_{0}-a\right)\right\}
$$

where $\vec{l}\left(x_{0}\right)$ is the tangent direction to the $C^{1}$ curve containing $x_{0}$ and the corresponding $\mathcal{L}_{j}$ in $\mathcal{L}$, and such that

$$
\int_{\mathcal{L}}|\theta|^{\frac{3}{4}} d \mathcal{H}^{1}<+\infty
$$

This characterization of the sequentially weak closure of smooth map in $W^{1,3}$ and the appearance of this intriguing exponent $3 / 4$ has stimulated the introduction the Sobolev critical exponents of rational homotopy groups .

\section{Existence of Rectifiable Poincaré Duals of topological SINGULARITIES OF MAPS IN SOBOLEV SPACES BETWEEN MANIFOLDS.}

Let $N^{n}$ be an arbitrary closed simply connected manifold. Let $p \in \mathbb{N}$ and let $z$ be an element of $\left(\pi_{p}\left(N^{n}\right) \otimes \mathbb{R}\right)^{*}$, (i.e. homomorphism from $\pi_{p}\left(N^{n}\right)$ into $\left.\mathbb{R}\right)$. $z$ simply extends to general $p$ and $N$ the topological degree for $N=S^{p}$ or the Hopf degree for $p=4 q-1$ and $N=S^{2 q}$.

For $u$ in $W^{1, p}\left(B^{p+1}, N^{n}\right)$ we introduce the measurable map

$$
\Phi_{z, u}: B^{p+1} \times \mathbb{R}_{+} \longrightarrow \mathbb{R}
$$

$$
(a, r) \longrightarrow z\left(\left[u_{\left.\right|_{\partial B_{r}^{p+1}(a)}}\right]\right)
$$

We introduce the following definition

Definition III.1. We say that a map $u$ in $W^{1, p}\left(B^{p+1}, N^{n}\right)$ has no z-type singularties if

$$
\Phi_{z, u} \equiv 0
$$

The following result generalizes theorem II.6 for $\pi_{2}\left(S^{2}\right)$ and theorem II.9 for $\pi_{3}\left(S^{2}\right)$ to general $\pi_{p}(N)$ :

Theorem III.1. [HR3] Let $N^{n}$ be an arbitrary closed simply connected manifold. Let $p \in \mathbb{N}$ and let $z$ be an element of $\left(\pi_{p}\left(N^{n}\right) \otimes \mathbb{R}\right)^{*}$. Let now $u$ in $W^{1, p}\left(B^{p+1}, N\right)$. Assume that $u$ is weakly approximable by maps without $z$-type singularities, then $\Phi_{z, u}$ admits a rectifiable Poincaré dual in the following sense: there exists a countable union $\mathcal{L}=\cup_{j} \mathcal{L}_{j}$ of disjoint $\mathcal{H}^{1}$-measurable subsets of $C^{1}$ oriented curves and an $\mathcal{H}^{1}$ measurable "multiplicity map" $\theta$ from $\mathcal{L}$ into $z\left(\pi_{p}\left(N^{n}\right)\right) \subset \mathbb{R}$ satisfying for every a in $B^{p+1}$ and almost every $r \in(0,1-|a|)$

$$
\Phi_{z, u}(a, r)=z\left(\left[u_{\left.\right|_{\partial B_{r}(a)}}\right]\right)=\sum_{x_{0} \in \partial B_{r}(a) \cap \mathcal{L}} \theta\left(x_{0}\right) \operatorname{sgn}\left\{\vec{l}\left(x_{0}\right) \cdot\left(x_{0}-a\right)\right\}
$$


where $\vec{l}\left(x_{0}\right)$ is the tangent direction to the $C^{1}$ curve containing $x_{0}$ and the corresponding $\mathcal{L}_{j}$ in $\mathcal{L}$, and such that

$$
\int_{\mathcal{L}}|\theta|^{\frac{p}{p+\nu z}} d \mathcal{H}^{1}<+\infty
$$

where $\nu_{z}$ is an integer depending only on $z$, the algebraic Sobolev critical exponents of $z$ defined below. If $\nu_{z}$ equals the analytic Sobolev critical exponents $\mu_{z}$ of $z$ defined below, then the existence of $\mathcal{L}$ and $\theta$ satisfying (III.3) and (III.4) is a necessary and sufficient condition for the weak approximability of a map $u$ in $W^{1, p}\left(B^{p+1}, N\right)$ by maps having no $z$-type singularities.

\section{The algebraic And analytic Sobolev CRitical exponents of A CLASS $z \operatorname{IN}\left(\pi_{p}\left(N^{n}\right) \otimes \mathbb{R}\right)^{*}$.}

Starting from the minimal model construction of Sullivan [Sul], Novikov in [Nov1], [Nov2], [Nov3] established integral formulations of the action of every element $z$ in $\left(\pi_{p}\left(N^{n}\right) \otimes \mathbb{R}\right)^{*}$ on maps $u$ from $S^{n}$ into $N^{n}$. This integral formulations are represented in [HR3] by formal linear combination of finite connected, simply connected oriented graphs of closed forms

$$
K_{z}=\sum_{l} \lambda_{l} K_{l}
$$

where $\lambda_{l} \in \mathbb{R}$ and each $K_{l}$ is a simply connected graph such that a closed differential form $\omega_{i}$ in $\wedge^{*} N$ and a variable $x_{i}$ in $S^{p}$ are assigned to each node. Moreover, to each segment connecting two nodes $i$ and $j$, with variables $x_{i}$ and $x_{j}$ and closed forms $\omega_{i}$ and $\omega_{j}$, the segment being oriented from $i$ to $j$, we assign a fixed "propagator form" $\alpha$ in $\wedge_{x_{i}}^{*} S^{p} \wedge_{x_{j}} S^{p}$ corresponding to the operation $d^{-1}$ (i.e. Precisely we take for $\alpha$ the Kernel corresponding to the non-local operation $d^{*} \Delta^{-1}$ ) : For every $\Omega$ in $\wedge^{l} S^{p}$ satisfying $d \Omega=0$, the $l-1$-form given by

satisfies

$$
\eta\left(x_{i}\right):=\int_{S^{p}} \alpha_{l}\left(x_{i}, x_{j}\right) \wedge \Omega\left(x_{j}\right)
$$

$$
d \eta=\Omega
$$

In order to simplify the notation we will omit the subscript $l$ for $\alpha$. Finally the following dimension condition is satisfied for each graph $K_{l}$ arising in the representation of $z$

(IV.5) $\quad p=\sum_{\text {node in } K_{l}}$ order of $\omega_{i}-$ number of branches in $K_{l}$.

Observe that because of the simply connectedness of each $K_{l}$ the number of nodes equals the number of branches +1 . 

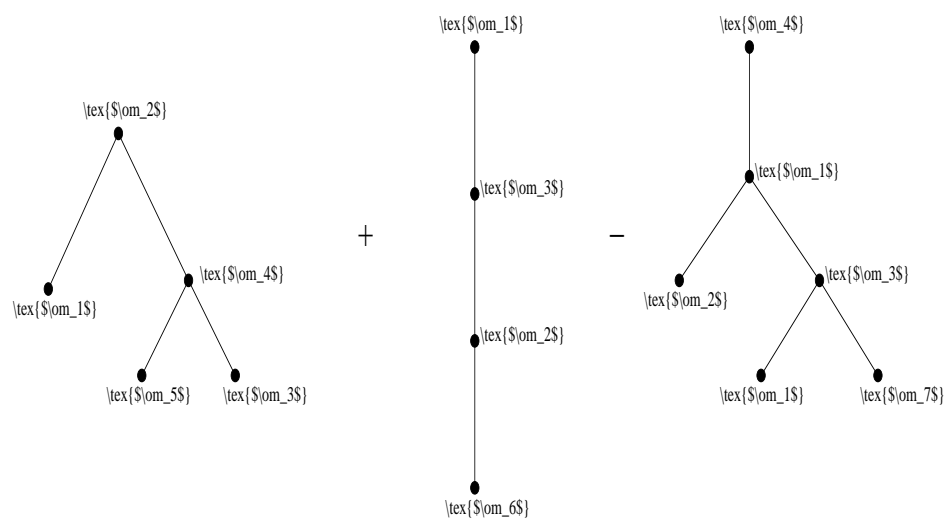

$\mid \operatorname{tex}\left\{\$ K_{-} \mid \$\right\}$

$\mid \operatorname{tex}\left\{\$ K_{-} 3 \$\right\}$

FiguRE 2. A formal linear combination of finite simply connected graphs of closed form of $\wedge^{*} N$.

Let now $u$ in $C^{1}\left(S^{p}, N\right)$ and denote $n_{K_{l}}$ the number of nodes in the graph $K_{l}$. Denote also $u^{\otimes n_{K_{l}}}$ the following map from $S^{p} \times \cdots \times S^{p}$ ( $n_{K_{l}}$ times) into $N \times \cdots \times N\left(n_{K_{l}}\right.$ times $)$ given by

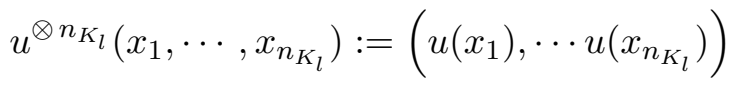

Using now this notation we introduce the following $p$-form on $S^{p}$

$$
u^{K_{l}}\left(x_{1}\right)=\int_{S^{p}} \cdots \int_{S^{p}}\left(u^{\otimes n_{K_{l}}}\right)^{*} \omega_{1} \wedge \cdots \omega_{n_{K_{l}}} \wedge \prod_{\{(i, j) \in I\}} \alpha\left(x_{i}, x_{j}\right)
$$

where the dots denote $n_{K_{l}}-1$ integrations on $S^{p}$, where $x_{1}$ denotes the variable at the summit of the graph $K_{l}$ and $I$ is the set of couples of nodes connected by an oriented segment in the graph $K_{l}$. With these notation the Novikov integral expression of $z([u])$ is

$$
z([u])=\int_{S^{p}} \sum_{l} \lambda_{l} u^{K_{l}} .
$$

We introduced in [HR3] the following definition

Definition IV.2. Let $z$ be an Homomorphism from $\pi_{p}\left(N^{n}\right)$ into $\mathbb{R}$ where $N^{n}$ is a closed simply connected manifold. We define the algebraic Sobolev critical exponent $\nu_{z}$ of $z$ as being the minimal integer among all formal linear combination of simply connected graphs $K_{z}=\sum_{l} \lambda_{l} K_{l}$ representing $z$ that one obtains by taking the maximal number of segments in $K_{l}$

$$
\nu_{z}:=\inf _{K} \max _{l}\left\{n_{K_{l}}\right\}-1 .
$$


The following proposition holds true

Proposition IV.1. Let $z$ be an element in $\left(\pi_{p}\left(N^{n}\right) \otimes \mathbb{R}\right)^{*}$ then there exists a constant $C_{z}$, depending only on $z$, such that

$$
z(u) \leq C_{z}\left[\int_{S^{p}}|\nabla u|^{p}\right]^{\frac{p+\nu_{z}}{p}} .
$$

the previous proposition motivates the following definition

Definition IV.3. Let $z$ be an Homomorphism from $\pi_{p}\left(N^{n}\right)$ into $\mathbb{R}$ where $N^{n}$ is a closed simply connected manifold. We define the analytic Sobolev critical exponent $\mu_{z}$ of $z$ as being the following limit

$$
\mu_{z}:=p \liminf _{z(u) \rightarrow+\infty} \frac{\log z(u)}{\log \int_{S^{p}}|\nabla u|^{p}}-p
$$

Observe that proposition IV.1 simply says that $\mu_{z} \leq \nu_{z}$.

Open problem : For which $z$ do we have $\mu_{z}=\nu_{z}$ ?

A similar question was adressed by M.Gromov in [Gr1], [Gr2] where Sobolev norms are replaced by Lipschitz norms. In fact no example is known where $\mu_{z}<\nu_{z}$. A form $z$ being given the computation of $\nu_{z}$ follows Novikov's construction of the integral representation of forms on rational homotopy groups and is hence algorithmic. $\mu_{z}$ however seems much more intricate to obtain and only few exemples are known where $\mu_{z}$ has been computed explicitely. In particular the case of the spheres is completely solved in [Ri1]

Theorem IV.2. [Ri1] For every $p>1$ integer and for every $z \in\left(\pi_{p}\left(S^{p}\right) \otimes \mathbb{R}\right)^{*} \simeq$ $\mathbb{R}, \nu_{z}=\mu_{z}=0$. For every positive integer $q$ and for every $z \in\left(\pi_{4 q-1}\left(S^{2 q}\right) \otimes \mathbb{R}\right)^{*} \simeq$ $\mathbb{R}, \nu_{z}=\mu_{z}=1$.

Finally we proved the following result.

Theorem IV.3. [HR3] For every closed simply connected 4-manifold $N^{4}$, for every integer $p>1$ and for every $z$ in $\left(\pi_{p}(N) \otimes \mathbb{R}\right)^{*}$,

$$
\mu_{z}=\nu_{z}
$$

\section{REFERENCES}

[ABL] F.Almgren, W. Browder, E.H.Lieb, Co-area, liquid crystals, and minimal surfaces. Partial differential equations (Tianjin, 1986), 1-22, Lecture Notes in Math., 1306, Springer, Berlin, 1988.

[Bet1] F. Bethuel, The approximation problem for Sobolev maps between two manifolds. Acta Math. 167(1991), no.3-4, 153-206.

[Bet2] Bethuel, F. A characterization of maps in $H^{1}\left(B^{3}, S^{2}\right)$ which can be approximated by smooth maps. Ann. Inst. H. Poincaré Anal. Non Linéaire 7 (1990), no. 4, 269-286. 
[BBC] F. Bethuel, H. Brézis, and J.-M. Coron, Relaxed energies for harmonic maps. Variational methods, 37-52, Progr. Nonlin. Diff. Eqns. Appl.,4, Birkhuüser Boston 1990.

$[\mathrm{BBH}]$ F.Bethuel, H.Brézis and F.Hélein Asymptotics for the minimization of a GinzburgLandau functional. Calc. Var. P.D.E. 1 (1993), no. 2, 123-148.

[BeZ] Bethuel, Fabrice; Zheng, Xiao Min Density of smooth functions between two manifolds in Sobolev spaces. J. Funct. Anal. 80 (1988), no. 1, 60-75.

[BCDH] Bethuel, F.; Coron, J.-M.; Demengel, F.; Hélein, F. A cohomological criterion for density of smooth maps in Sobolev spaces between two manifolds. Nematics (Orsay, 1990), 15-23, NATO Adv. Sci. Inst. Ser. C Math. Phys. Sci., 332, Kluwer Acad. Publ., Dordrecht, 1991.

[BCL] H. Brézis, J.-M. Coron, and E. Lieb, Harmonic maps with defects. Comm. Math. Phys. 107(1986), no.4, 649-705.

[BrN1] Brézis, H.; Nirenberg, L. Degree theory and BMO. I. Compact manifolds without boundaries. Selecta Math. (N.S.) 1 (1995), no. 2, 197-263.

[BrN2] Brézis, Haim; Nirenberg, Louis Degree theory and BMO. II. Compact manifolds with boundaries. With an appendix by the authors and Petru Mironescu. Selecta Math. (N.S.) 2 (1996), no. 3, 309-368.

[Fe] H.Federer Geometric measure theory Springer 1969.

[Fe] Federer, Herbert Geometric measure theory. Die Grundlehren der mathematischen Wissenschaften, Band 153 Springer-Verlag New York Inc., New York 1969.

[GMS] M.Giaquinta, G.Modica and J.Soucek Cartesian Currents in the Calculus of Variations vol. I and II, Springer (1998).

[GiM] Giaquinta, M.; Mucci, D. On sequences of maps into a manifold with equibounded $W^{1 / 2}$. energies. J. Funct. Anal. 225 (2005), no. 1, 94-146.

[GM] P.A.Griffiths and J.W.Morgan Rational Homotopy Theory and Differential Forms Progress in Math. 16, Birkhaüser (1981).

[Gr1] M.Gromov Metric structures for Riemannian and non-Riemannian spaces Progress in Math. 152, Birkhäuser, (1999).

[Gr2] M.Gromov Quantitative Homotopy Theory in Prospects in Mathematics, Hugo Rossi Ed., A.M.S., (1998).

[Haj] Hajłasz, Piotr "Approximation of Sobolev mappings". Nonlinear Anal. 22 (1994), no. 12, 1579-1591.

[Han] Hang, Fengbo Density problems for $W^{1,1}(M, N)$. Comm. Pure Appl. Math. 55 (2002), no. 7, 937-947.

[HaL1] F.Hang and F.H.Lin Topology of Sobolev mappings. Math. Res. Lett. 8 (2001), no. 3, $321-330$

[HaL2] F.Hang and F.H.Lin Topology of Sobolev mappings. II. Acta Math. 191 (2003), no. 1, $55-107$.

[HaL3] Hang, Fengbo; Lin, Fanghua Topology of Sobolev mappings. III. Comm. Pure Appl. Math. 56 (2003), no. 10, 1383-1415.

[HR1] R.Hardt and T.Rivière Connecting topological Hopf Singularities Ann. Scuoa. Norma. Sup. Pisa (5) Vol. II (2003), pp. 287-344.

[HR2] Hardt, Robert; Rivière, Tristan Ensembles singuliers topologiques dans les espaces fonctionnels entre vari?t?s. (French) [Topological singular sets in functional spaces between manifolds] Séminaire: équations aux Dérivées Partielles, 2000-2001, Exp. No. VII, 14 pp., Sémin. équ. Dériv. Partielles, Ecole Polytech., Palaiseau, 2001.

[HR3] R.Hardt and T.Rivière Connecting rational homotopy type singularities. to appear in Acta Math. (2007).

[HR4] R.Hardt and T.Rivière Weak density of smooth maps in $W^{1, p}\left(B^{n}, S^{p-1}\right)$, the stable case $p>4$. in preparation. 
[Nov1] S.P.Novikov The analytic generalized Hopf invariant. Many-valued functionals. Russian Math. Surveys, 39 (1984), 113-124.

[Nov2] S.P.Novikov Analytic Homotopy Theory. Rigidity of Homotopy Integrals Soviet Math. Dokl., 32 (1985), 285-288.

[Nov3] S.P.Novikov Analytical theory of homotopy groups, Lect. Notes in Math. 1346, Springer, 99-112.

[Pak] Pakzad, Mohammad Reza Weak density of smooth maps in $W^{1,1}(M, N)$ for non-abelian $\pi_{1}(N)$. Ann. Global Anal. Geom. 23 (2003), no. 1, 1-12

[PaR] Pakzad, M. R.; Rivière, T. Weak density of smooth maps for the Dirichlet energy between manifolds. Geom. Funct. Anal. 13 (2003), no. 1, 223-257.

[Ri1] T.Rivière Minimizing fibrations and p-harmonic maps in homotopy classes from $S^{3}$ into $S^{2}$ Comm. Anal. Geom., 6 (1998), 427-483.

[Ri2] Rivière, Tristan Dense subsets of $H^{1 / 2}\left(S^{2}, S^{1}\right)$. Ann. Global Anal. Geom. 18 (2000), no. $5,517-528$.

[SaU] Sacks, J.; Uhlenbeck, K. The existence of minimal immersions of 2-spheres. Ann. of Math. (2) 113 (1981), no. 1, 1-24.

[ScU] R.Schoen and K.Uhlenbeck, Approximation theorems for Sobolev mappings preprint (1984).

[St] E.M.Stein Singular integrals and differentiability properties of functions Princeton University Press, 1970.

[Sul] D.Sullivan Infinitesimal computations in topology Inst. Hautes Études Sci. Publ. Math., 47 (1977), 269-331.

[Wh] B.White Homotopy classes in Sobolev spaces and the existence of energy minimizing maps Acta. Math., 160 (1988), 1-17.

Tristan Rivière

Department of Mathematics

ETH Zentrum, CH-8093 Zürich, Switzerland.

Email: riviere@math.ethz.ch 